\title{
Process mining on machine event logs for profiling abnormal behavior and root cause analysis
}

\author{
Jonas Maeyens • Annemie Vorstermans • \\ Mathias Verbeke
}

Received: date / Accepted: date

\begin{abstract}
Process mining is a set of techniques in the field of process management that have primarily been used to analyse business processes, for example for the optimization of enterprise resources. In this research, the feasibility of using process mining techniques for the analysis of event data from machine logs is investigated. A novel methodology, based on process mining, for profiling abnormal machine behavior is proposed. Firstly, a process model is constructed from the event logs of the healthy machines. This model can subsequently be used as a benchmark to compare process models of other machines by means of conformance checking. This comparison results in a set of conformance scores related to the structure of the model and other more complex aspects such as the differences in duration of particular traces, the time spent in individual events, the relative path frequency, etc. The identified differences can subsequently be used as a basis for root cause analysis. The proposed approach is evaluated on a real-world industrial data set from the renewable energy domain, more specifically event logs of a fleet of inverters from several solar plants.
\end{abstract}

Keywords Process mining · Event $\operatorname{logs}$ · Industrial machinery · Irregular behavior · Profiling · Root cause analysis

J. Maeyens · A. Vorstermans

KU Leuven - Technology Campus Ghent

Gebroeders de Smetstraat 1

B-9000 Ghent

E-mail: jonas.maeyens@gmail.com,annemie.vorstermans@cs.kuleuven.be

M. Verbeke

Sirris - Data and AI Competence Lab

Bd. A. Reyerslaan 80

B-1030 Brussels

E-mail: mathias.verbeke@sirris.be 


\section{Introduction}

Internet-of-Things (IoT) technology increasingly finds its way to the industrial domain, and as most IT-related phenomena, the growth of (event) data complies with Moore's Law 3. Many organizations now realize that the goal is not to collect as much data as possible, but to use the gathered data to gain valuable insights, to improve current processes and to enhance overall efficiency. Furthermore, industrial machines are being equipped with sensors, capturing the machine's states in the form of raw data, which are then sent to information systems. These sensor data are then stored in data logs that allow the monitoring and the analysis of the functioning of the machines. However, it is possible that not only raw time series data but also events are being logged. These events are often correlated to a certain pattern in the sensor data.

A process is a set of discrete activities or events that are performed in order to achieve a particular goal. Process mining is a family of techniques used for extracting insights from these processes by the means of analyzing the event data that is generated during the execution of the process. The end goal of process mining is to model, monitor, and optimize the underlying processes. It emerged as a new scientific discipline, situated on the crossroads between conventional Business Process Management on the one hand, which mostly focuses on process models without considering the (event) data, and Data Mining on the other.

In this article, the feasibility of using process mining techniques for the analysis of machine log data is examined. A novel methodology for profiling irregular machine behavior is proposed. Firstly, a process model is constructed from the event logs of the healthy machines, by means of a process discovery algorithm. This model can subsequently be used as a benchmark to compare behavior of other machines utilizing conformance checking [11. This comparison results in a set of fitness scores, indicating how well a certain process execution can be aligned with the healthy process model. These fitness scores can then be analyzed for the profiling of abnormal machine behavior. These differences can also be visualized (interactively) on the process model itself, which serves as the basis for root cause analysis. This is a systematic process for identifying the primary cause of a particular problem with an industrial asset [15. It assumes that events are interrelated and that by tracing back the sequence it is possible to discover where the problem started and how it grew into the faced issue.

The methodology is validated through a case study in the renewable energy domain, using a dataset of event logs of a fleet of inverters transforming currents from DC to AC in a photovoltaic (PV) plant. More specifically, the profiling of irregular machine behavior includes the identification and the prediction of outages of these inverters (e.g., caused by a grid failure or disconnected strings), based on a decrease in fitness scores.

This article is structured as follows. Section 2 gives an overview of related research. In Section 3 the methodology is described on a conceptual level. 
Then in more detail, Section 4 will briefly discuss the data from the photovoltaic plant, and accordingly some data preparation actions. In Section 5 the discovery of a healthy process model that describes the functioning of a healthy inverter will be described. This healthy process model will then be used to detect irregular inverter behaviour in Section 6 .

\section{Related work}

Process mining is a set of techniques in the field of process management that until now have primarily been used to analyse business processes, for example for the optimization of enterprise resources based on the analysis of transaction logs. The use of process mining for analysing event logs from industrial machinery, which is the scope of this work, is limited.

Some related work is available on the use of process mining for anomaly detection and root cause analysis in business processes. Calderón-Ruiz and Sepúlveda [5] present an approach to extract behavioral patterns from both sucessful and failed business process event logs using the Performance Sequence Diagram Analysis algorithm. Both pattern sets are compared considering control flow and time perspectives, in which the identified differences represent potential causes of failures in the respective business processes. Gupta et al. [8] propose Pariket, a multi-step process for identifying anomalous traces and executions from event logs generated by Process Aware Information Systems as well as a technique for root cause analysis of these anomalous traces. To this end, the event log is transformed into a sequential dataset and windowbased and Markovian techniques are applied to identify anomalies. Machine learning techniques such as decision tree classifiers are employed to extract rules explaining the root causes of anomalous transactions, in contrast to the approach proposed in this paper, which leverages the fitness scores resulting from conformance checking to detect and predict abnormal behavior. Suriadi et al. 12 propose a root cause analysis approach that enriches an event log with relevant contextual information for explaining possible root causes of risk incidents in business processes. This enriched log is subsequently transformed into a format such that it can be analyzed using established classification techniques. Consequently, the authors transform a process mining problem into a machine learning one. van der Aalst and de Medeiros [13] present an approach to detect anomalous process executions in audit trails. The technique is focussed on the security domain in a business process setting, which is different in characteristics than industrial machinery.

\section{Methodology}

This section will describe the methodology on a conceptual level. It can be divided into three parts: data preparation and exploration, process mining, and evaluation. 


\subsection{Data Preparation and Exploration}

The provided inverter data of the photovoltaic installation is not directly suitable for process mining. All events of all inverters were captured into a single file. Consequently, the event log first need to be split in a series of event logs per inverter in the photovoltaic plant, and transformed into the appropriate file format such that it can serve as input for a process mining tool like ProM. During this process of data preparation, the event log data are thoroughly explored.

\subsection{Process Mining}

As a result of the data preparation step, event logs are available for all the inverters (healthy and failing) in the PV plant. Due to computational power limitations, only a selection of healthy inverter event logs is subsequently used to discover a healthy process model. This healthy process model should describe the functioning of a healthy inverter.

However, the quality of this healthy process model needs to be verified. The process model can be validated using conformance checking techniques on both the training data and test data. Replay fitness is the quality criterion that is most often used in conformance checking, resulting in scores ranging between 0 and 1 indicating how well (parts of) an event log fits in the healthy process model. The replay fitness, or fitness in brief, reflects to what extent the model is able to play back the served behavior. A model with perfect replay fitness is able to play all traces within the event log. Replay fitness can be defined at the level of events (which fraction of the events occur in the model), as well as at the level of cases (the fraction of traces that can be mimicked by the model). In this work, the alignment method for calculating event log fitness and the corresponding fitness score as defined in [1] will be used.

In this case, a fitness score of 0 indicates that the training and test event data do not fit in the healthy process model and is thus indicating poor quality, whereas a fitness score equal to 1 indicates a perfect replay fitness, thus meaning the training and test event data can be described perfectly by the healthy process model.

\subsection{Evaluation}

In a final step, the healthy process model can be used to profile irregular inverter behavior. More specifically, the healthy process model is used as a benchmark to compare event logs of failing inverters with the healthy process model using conformance checking. This benchmarking results in a set of fitness scores which, subsequently, are analyzed to identify and predict outages. 


\subsection{Process mining framework}

In this article, process mining techniques offered by ProM ${ }^{1} 7$ and EDUProM $^{2}$ are used. ProM is a generic open source framework that supports a wide variety of process mining techniques in the form of plug-ins. It is considered state-ofthe-art as novel process mining research results are rapidly integrated in the framework. EDUProM is a library for working with ProM capabilities in a non-interactive way, allowing one to automate the workflow without manual intervention.

\section{Data preparation and exploration}

This section describes the process of data preparation, as well as a comparison between healthy and failing event log data.

In the photovoltaic plant, sensors are logging the levels of irradiation and the corresponding amount of generated $A C$ power for each inverter. Next to these data, events are being logged in a separate file. For example, these events can indicate that a certain inverter stopped working, or that it re-calibrated its sensors. Also, knowledge about which inverters are functioning correctly (= healthy inverters) and which inverters are not (= failing inverters) is available. For each of the inverters, there is a file available with the points in time at which an outage was registered and how long the outage lasted.

More concrete, one file contains all the event data of 16 healthy inverters. Similarly, another file contains all the event data of 26 failing inverters. As mentioned before, these event log data first need to be prepared in order to perform process mining.

\subsection{Data Preparation}

More specifically, for process discovery (i.e. the discovery of a process model, based on event log data), the following attributes need to be present in the event log: timestamp, event ID, trace ID. The timestamp describes when the event occurred, while the event ID uniquely identifies which event occurred. Each event in the event log belongs to a specific trace (indicated by the trace ID). A trace captures a single process cycle as an ordered sequence of events.

The data preparation process can be described as follows:

1. Splitting of the event log data. For each inverter, a separate file (event log) was generated.

2. Removal of empty records: for some timestamps, no events were registered.

3. Addition of a trace ID for each event. The trace ID starts at 0 for the first trace. While iterating over all of the events in the event log of a certain

\footnotetext{
1 http://www.processmining.org/prom/ http://www.promtools.org

2 https://github.com/yosid16/edu-prom
} 
Table 1 Event log of an inverter in the PV plant

\begin{tabular}{lll}
\hline timestamp & eventID & traceID \\
\hline 2015-01-04 16:13:45+00:00 & 1003.0 & trace 10 \\
2015-01-04 16:13:59+00:00 & 6.0 & trace 10 \\
2015-01-04 16:17:21+00:00 & 255.0 & trace 10 \\
2015-01-05 07:25:00+00:00 & 1.0 & trace 11 \\
2015-01-05 07:31:05+00:00 & 14.0 & trace 11 \\
2015-01-05 08:02:08+00:00 & 6.0 & trace 11 \\
\hline
\end{tabular}

inverter, all events are assigned the same trace ID until a 'stop' event is encountered. This stop event indicates the end of a process cycle (in this case a particular event type that indicates that the inverter was stopped), and thus the end of that trace. The trace counter is then incremented, after which it is assigned to the next event.

The result of this process of data preparation is that for every inverter (healthy or failing) in the PV plant, there is an event log available (example shown in Table 1) which describes the behavior of that inverter in a time span of two years. It is then possible to combine a selection of healthy inverter logs (6 healthy inverters). This is done by concatenating the event logs of individual inverters. Also, a selection of failing inverter logs (6 failing inverters) is combined into one event log. The selection of healthy inverters consists of inverters that had the least outages. Similarly, the selection of failing inverters consists of inverters that had the most outages. The resulting combined healthy event $\log$ then describes the events that can be observed when an inverter is functioning correctly, while the combined failing event log describes the events that can be observed when an inverter is malfunctioning. The resulting log files can then be processed at once to extract process models describing the prototypical behaviour of healthy and failing inverters respectively. The combined healthy event log contains 70750 events over a period of 2 years, while the combined failing event log contains 75479 events. This selection of healthy and failing inverters is necessary due to computational power limitations.

\subsection{Data exploration}

When importing both the combined healthy and failing event logs in ProM, it is possible to visualize remarkable differences in relative event frequency, event duration, transitions, etc. by using the Process Comparator plug-in [4]. By using this plug-in, it is possible to observe that certain events only occur in the combined failing event log (events 4, 110, 1006, 1023, 15, 1015, 1049, 1226, 1185, 1062, 1072, 1174, 1236, 1246, 1133, 1092, 1113, 1144, 1090, 1227, 1164, 1031 and 1052 are not present in the healthy event $\log$ ). Also, some events have a higher relative frequency in the failing event log than the same event in the healthy event log (e.g. event 14: $46.845 \%$ vs. $22.711 \%$ ).

A visual representation of the comparison between the combined healthy event $\log$ and the combined failing event $\log$ is depicted in Figure 1. To avoid 
non-significant differences, events that occured less than $3 \%$ in the event logs were not taken into account in the comparison, which furthermore limits the complexity of the model. The colors indicate the similarity between the two event logs, using Cohen's distance measure. The bluer colors indicate events and transitions that are characteristic for the healthy event logs, whereas the redder colors indicate events and transitions occurring more frequently in the combined event log of failing inverters. White events occur as frequently in both event logs, as illustrated by the start and stop events. Note that, in contrast to a process model, the structure of the model is of less importance here.

These differences may be correlated to the malfunctioning of an inverter, and may thus be useful to consider for a further root cause analysis (identifying the cause of an outage). For example, the transition from the start event to event 1 ('Wait Sun/Grid') is more prevalent for healthy inverters (97.24\%) than for failing inverters $(65.59 \%)$. For failing inverters, in a number of cases a 'Riso Low' error is encountered, which is usually caused by a low insulation resistance on the DC circuit connected to the inverter. This is useful information to pinpoint the primary cause of an outage during maintenance interventions.

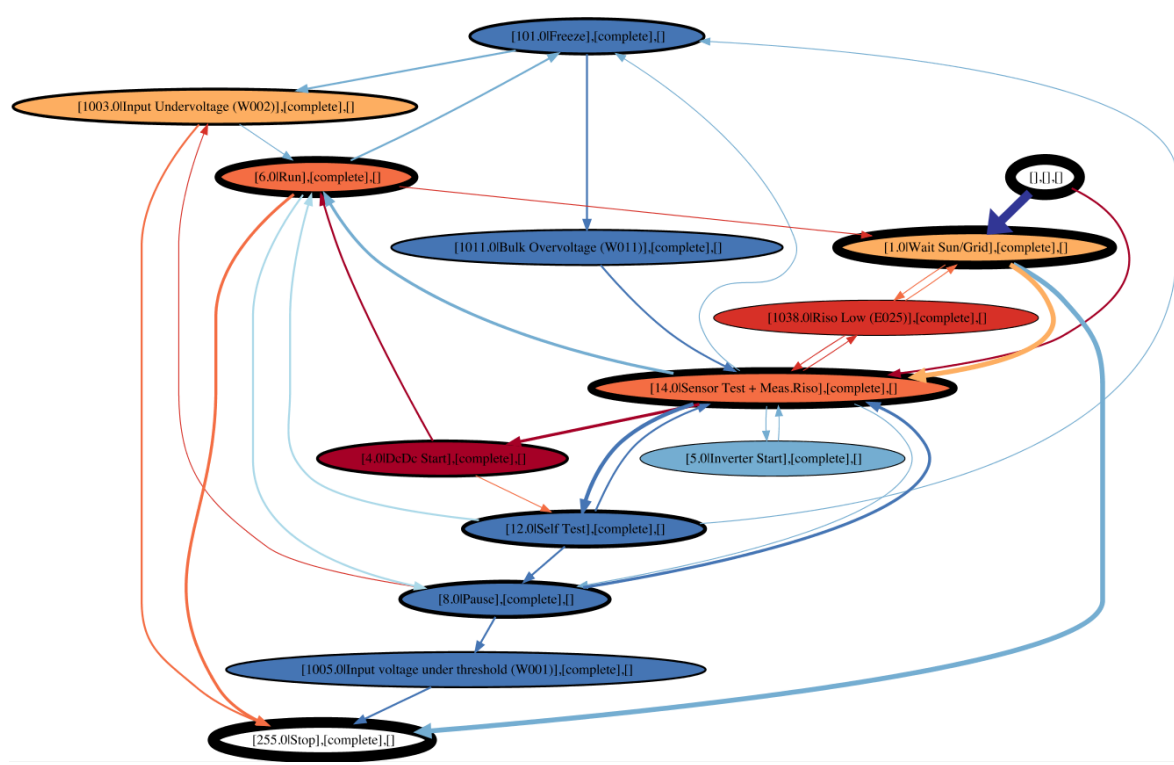

Fig. 1 Visualisation of the comparison between the combined healthy event log and the combined failing event log 


\section{Process mining}

As a result of the data preparation process, a combined healthy event log and a combined failing event log are generated. This healthy event log, describing the functioning of a healthy inverter, can then be used to discover a process model.

\subsection{Inductive Mining algorithm}

For the discovery of a healthy process model, the Inductive Mining (IM) algorithm is used [10]. This choice is motivated by the fact that the IM algorithm is always capable of generating correct process models, in contrast to other process discovery techniques such as the Heuristics Mining algorithm 14. Furthermore, the generated models are often limited in terms of complexity. In contrast to the Alpha algorithm [2, the IM algorithm is able to deal with short loops and events that are skipped in the event log.

To extract a process model, the IM algorithm first finds a prominent split in an event log. Subsequently it detects how these splits are related by identifying the so-called operator. It then repeats the aforementioned steps on both splitted logs. The result is a process model in the form of a process tree. In contrast to other representation methods, a process model that is represented as a process tree always satisfies the conditions of a sound model. Furthermore, a process model in this format has the advantage that it can be transformed to one of the other standards (e.g., Petri Net, BPMN), allowing an alternative view on the model, possibly yielding additional insights. In this work, the Petri Net notation will be used.

A downside of the basic IM algorithm is that it cannot handle incomplete data and that the frequencies with which traces or events occur are not taken into account during the generation of a process model. To deal with these issues, a number of more advanced IM algorithms have been proposed [9]. In this work, the Inductive Mining - infrequent variant will be used, as it uses several kinds of filtering with the goal to solely model the regular behavior.

\subsection{Process discovery in noisy event logs}

It is possible that some critical events (i.e. events leading to an outage) occur in the healthy event log. Since it is not desirable to take these erroneous behaviors into account for the discovery of a healthy process model, it is necessary to specify a noise threshold for filtering out these events. In this case, a noise threshold equal to 0.20 is set. This means that in the discovery of a process model, $20 \%$ of the least occurring events in the healthy event log are not considered. In Table 2, the fitness score is calculated for the training and test data with varying values of noise threshold. The test data consists of 3 healthy event logs. We can observe a decrease in fitness when the noise threshold is 
increased from 0.20 to 0.30 , meaning a loss in quality in comparison to the noise threshold equal to 0.20 . A noise threshold equal to 0 results in a perfect replay fitness, therefore no events were filtered from the event log.

A noise threshold equal to 0.20 produces fitness scores $>99 \%$, meaning the healthy event log fits the healthy process model almost perfectly while filtering out the infrequent (critical) events. Therefore, it can be concluded that the healthy process model is representative of the functioning of the healthy inverters in the photovoltaic plant.

Table 2 Replay fitness for different values of noise threshold

\begin{tabular}{|l|l|l|l|l|l|}
\hline Noise threshold & $0 \%$ & $5 \%$ & $10 \%$ & $20 \%$ & $30 \%$ \\
\hline Fitness training & 1 & 0.997590 & 0.991336 & 0.990846 & 0.970349 \\
\hline Fitness test & 1 & 0.997463 & 0.993350 & 0.991642 & 0.976704 \\
\hline
\end{tabular}

\subsection{Visual representation of the process model}

This process model is visualized in Figure 2 in Petri net notation. A Petri net is a two-part graph, consisting of transitions (denoted as white rectangles), which represent all the unique activities that can occur in the process. On the other hand, there are places (white circles) that connect the transitions. The evolution of the event log can be visualised as tokens flowing through the process model, in which the state of the process model is determined by the distribution of tokens over the Petri net. A transition is activated if there is a token in each of the input places. An input place is a place on the left side of a transition. Output places are located on the right side of the transition. When an activated transition is executed or fired, the tokens in the input places are moved to the output places. In this way a next transition can be activated. An exception are silent transitions. These transitions are not observed directly in the event log, but are derived from the behaviour described in the event log. Usually they are added by the process discovery algorithm to arrive at a correct process model. In Petri net notation, these transitions are denoted as black squares.

\section{Evaluation}

In this section, the healthy process model will be used to detect irregular inverter behavior. More specifically, the identification and prediction of outages through a decrease in fitness scores are explored. 


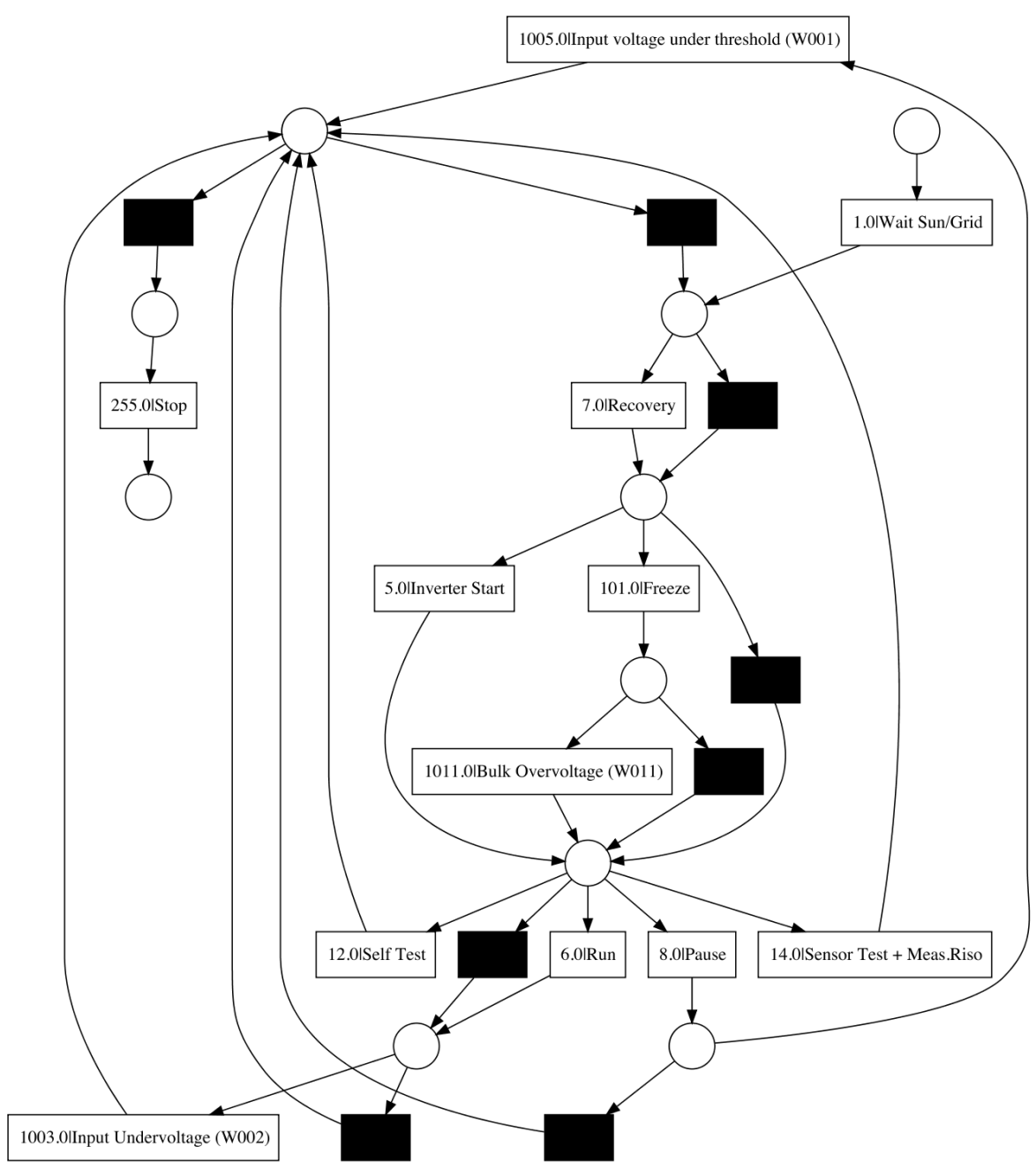

Fig. 2 Process model describing the functioning of a healthy inverter

\subsection{Outage identification}

Firstly, it should be possible to identify an outage by observing a decrease in the fitness score. We expect an inverter to be functioning (more or less) correctly until an outage occurs. Therefore, the majority of the events that were registered before an outage occurred, should fit in the healthy process model, resulting in a relatively high fitness score. On the other hand, we expect the events that where registered during an outage, to be critical events or at least irregular sequences of events, that do not fit in the healthy process model. Hence, the expectation is that the events which were registered during 
an outage will result in a lower fitness score when aligned with the healthy process model.

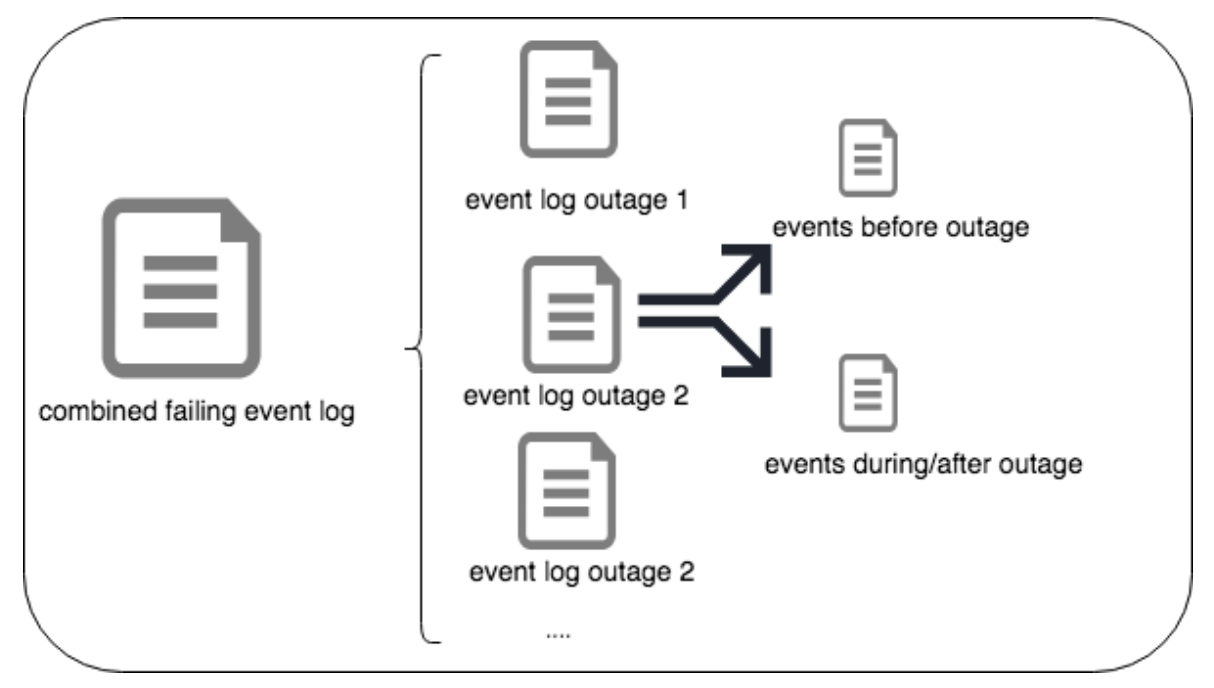

Fig. 3 Splitting the event log

To confirm our expectation, the combined failing event log is split into event logs per outage, which is possible given that the occurrences of the outages per inverter as well as their duration are known. Subsequently, each of these individual outage event logs are split into two parts, containing the events in the period before and after the outage (e.g., due to the occurrence of overvoltage of the inverter) respectively. Note that the latter sublog contains all events which were registered from the moment the outage was identified until the outage was resolved. This process of event log splitting is illustrated in Figure 3 .

Afterwards, each of the sub logs describing the behavior before and after the actual outage, can be aligned with the healthy process model. For this alignment we consider the periods before and after the outage separately. Hence, for each sub log, two fitness scores are calculated. The first fitness score (i.e. the fitness before outage) indicates how well the part of the sub log before the outage can be aligned with the healthy process model. The second fitness score (i.e. the fitness score after the outage) indicates how well the part of the sub log after the outage can be aligned with the healthy process model. This alignment, resulting in two fitness scores, is calculated using conformance checking. A fitness score always lies between 0 and 1 , where a fitness score of 0 indicates that (the part of) the event log does not fit in the process model at all (i.e. unfitting), while a fitness score of 1 indicates that (the part of) the event $\log$ fits perfectly in the process model (i.e. perfectly fitting). Since this 
Table 3 Fitness scores for the periods before and after the outages

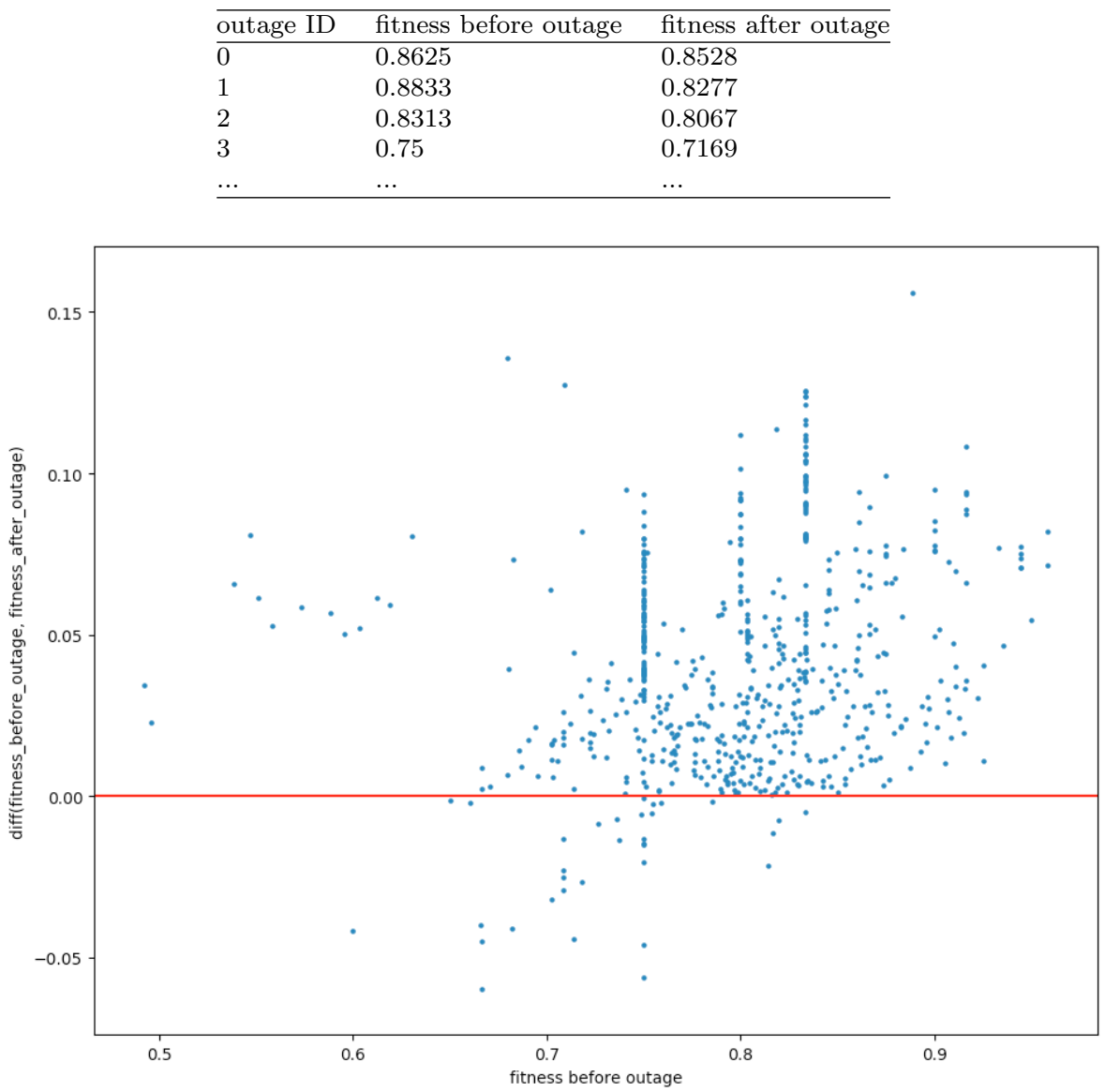

Fig. 4 Differences in fitness scores before and after the outage

conformance checking requires a lot of repetition (for each sub log, two fitness scores need to be calculated), EDUProM is used.

In the selected data, 629 outages occurred, spread over a total of 6 failing inverters. Therefore, 2 × 629 fitness scores were calculated. The results can be represented in a table such as Table 3, where each row represents one sub log. For the results shown in Table 3 , it is possible to observe a decrease in the fitness scores after the outages. This is due to the fact that in this state the inverter is operating in a failure mode which needs to be resolved before the inverter goes back to normal operation, and consequently is less compliant with process model of an inverter functioning normally.

The scatter plot in Figure 4 represents the results for all the outages. Here, the differences in fitness scores before and after each outage are visualized, with on the y-axis the difference in fitness scores (i.e. fitness before outage - fitness 
after outage) and on the $\mathrm{x}$-axis the fitness score before outage. This means that for all of the (positive) differences above the red line, we can observe a decrease in fitness score. All the (negative) differences under the red line in the scatter plot indicate an increase in fitness score. The scatter plot proves that the majority of differences lies above the red line and therefore a decrease in fitness score is observed. When quantifying these results, we observe that in $96.044 \%$ of the outages, an occurrence of an outage is correlated with a decrease in fitness score.

However, in some cases, it is possible to observe an increase in fitness $(3.956 \%)$. Also, it is remarkable that for $4.451 \%$ of the outages, the fitness before the outage is lower than $70 \%$. These cases need to be further examined, as they may indicate irregular behavior prior to the occurrence of an outage. For the identification of an outage by means of a decrease in fitness score, it is safer to consider the cases with a fitness before outage $<0.70$ as outliers.

Therefore, it is more correct to conclude that an outage can be identified through a decrease in fitness score with an accuracy of $96.416 \%$, if the fitness score before the outage is higher than $70 \%$.

\subsection{Outage prediction}

In the previous section, the possibility of identifying an outage through a decrease in fitness scores was explored. For the majority of outages, a decrease in fitness scores was indeed observed. In some cases however, a relatively low fitness score before outage was observed. In this section, we examine if irregular inverter behavior can be observed prior to an actual outage. Hence, this irregular behavior is not a critical behavior and therefore does not immediately result in an outage. However, it may indicate that an outage will occur at a later point. The goal is to profile this irregular behavior through a decreasing fitness score, in the run up to the actual outage. Practically, only the events that occurred before each outage are considered (i.e. the events before outage in Figure 3p. Using this event log before the outage, several sub logs are generated according to the process described in Figure 5 Each sub log period before outage describes a period in the run up to the outage. Period 1 before the outage only contains one trace, which is the first trace prior to the outage. Period 2 before the outage then consists of 2 traces: the first and second trace. Subsequently, each additional period before the outage contains one additional trace until the last period before outage, i.e. period $m$ before outage, contains all the traces (and therefore all the events) before the outage.

As a result, for each outage, there is a series of event logs (periods before outage) available increasing in size, in the run up to the actual outage. As each event $\log$ before the outage differs in length, a different number of periods before the outage is generated for each outage. Fitness scores can then be calculated for each period before the outage in a certain series. The evolution of the fitness scores for a particular outage is represented by Table 4 . 


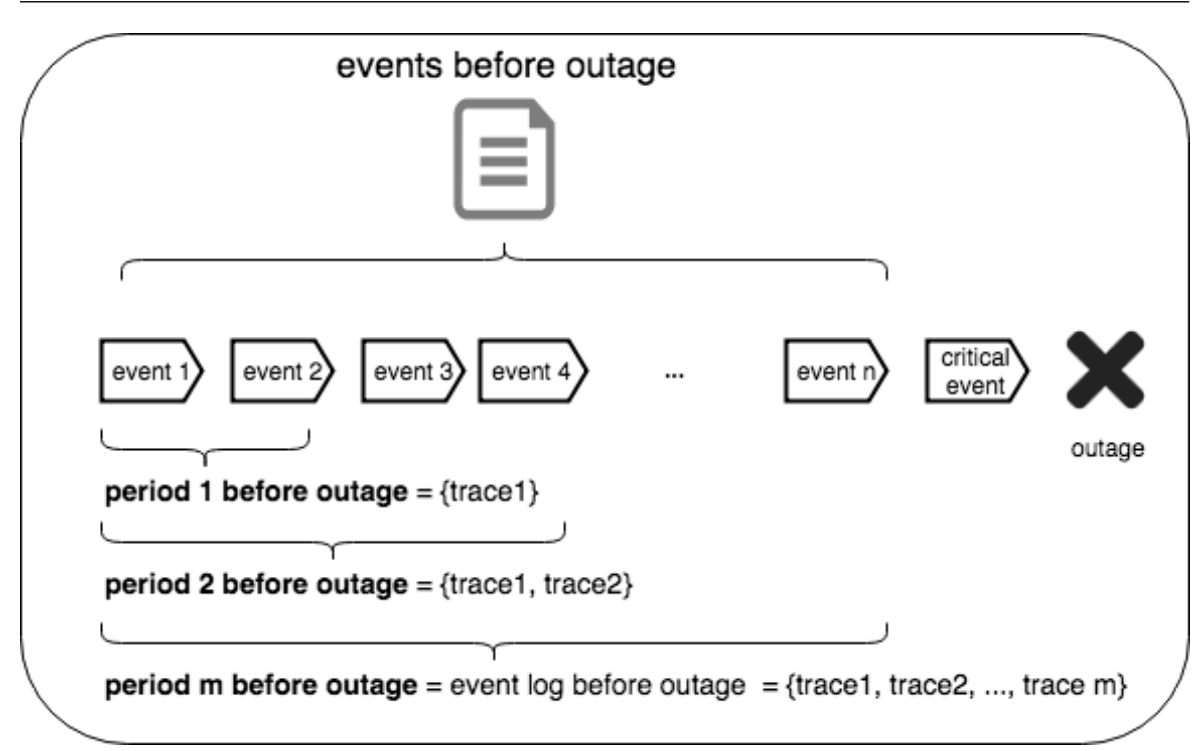

Fig. 5 Event logs in the run up to an outage

Table 4 Evolution of fitness scores in the run up to an outage

\begin{tabular}{ll}
\hline index period before outage & fitness \\
\hline 0 & 0.8571428571428572 \\
1 & 0.8035714285714286 \\
2 & 0.8214285714285715 \\
3 & 0.8303571428571429 \\
$\ldots$ & $\ldots$ \\
\hline
\end{tabular}

Table 5 Fitness evolution matrix in the run up to an outage

\begin{tabular}{llllll}
\hline fitness $\backslash$ PBO & period0 & period1 & period2 & period3 & period4 \\
\hline $1-0.9$ & 2 & 17 & 10 & 8 & 5 \\
$0.9-0.85$ & 71 & 41 & 42 & 48 & 52 \\
$0.85-0.80$ & 89 & 71 & 74 & 71 & 68 \\
$0.80-0.75$ & 5 & 30 & 40 & 42 & 38 \\
$0.75-0.70$ & 0 & 19 & 11 & 9 & 15 \\
\hline
\end{tabular}

Here, the row with index 0 represents the fitness score for period 0 before outage (containing only one trace). Row 2 then represents the fitness score for period 2 before outage, and so on for all periods before outages. This particular example confirms the expectation of decreasing fitness due to irregular behavior.

In order to visualize the general trend in the evolution of fitness scores for all of the outages, 5 fitness scores were selected out of each series of fitness scores. Here, period 0 always represents period 0 before outage, thus containing only one trace. Period 4 always represents the whole event log before the outage, therefore containing all events that were registered before outage. Pe- 


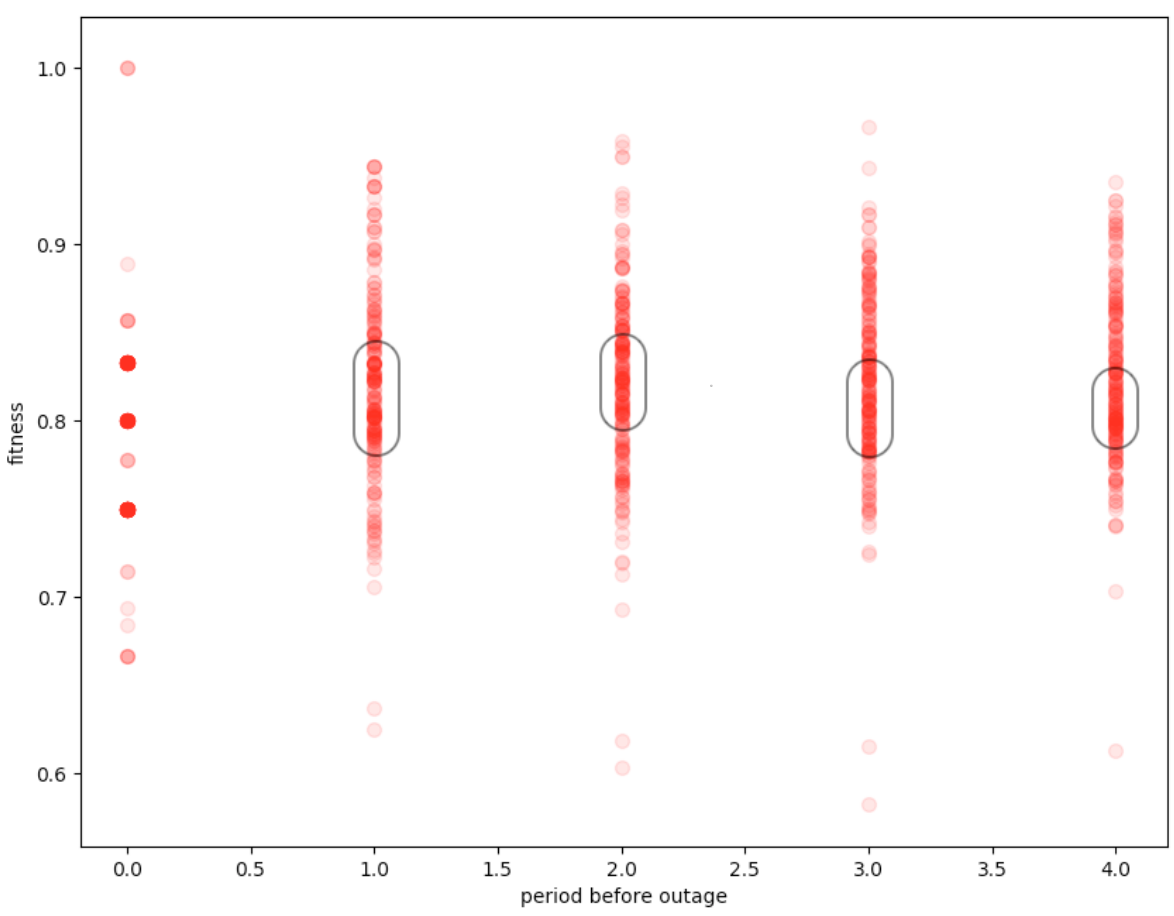

Fig. 6 General trend in evolution of fitness scores in the run up to an outage

riods 1,2 and 3 then represent periods between period 0 and 4 . The scatter plot in Figure 6 visualizes this trend. Table 5 can be considered as the numerical representation of this scatter plot. We can observe that for period 0 , containing only one trace, the majority of the concentration has a fitness score in between 0.80 and 0.90 . Subsequently, for period 1, 2 and 3, the concentration shifts slightly downwards in the scatter plot (the darker parts indicate a more dense area of concentration). In the evolution matrix, we can confirm this by observing a majority of cases that have a fitness score in the range of $0.80-0.85$.

When considering the trend across periods, a slight decrease in fitness scores can be observed in the run up to an outage, therefore indicating the occurrence of irregular behavior prior to the outage.

\section{Conclusions}

The goal of this article was to explore the feasibility of applying process mining techniques in an industrial context, more specifically for profiling abnormal behavior and root cause analysis in machine event logs. We can conclude that the initial findings prove process mining techniques to be meaningful. 
Existing techniques in the PV domain primarily focus on the raw sensor data for error detection, while the use of process mining and event log data is relatively new 6. Process mining offers an added value in interpretability of processes and models. Additionally, process mining allows for an accurate comparison of process models.

In this use case, profiling abnormal machine behavior included the possibility to identify and detect inverter outages. The results prove that an outage can be identified by means of a decrease in fitness. Also, we observed a slight decrease in fitness scores prior to an outage, therefore it is possible to identify irregular behavior in the run up to an outage. However, further validation of the evolution in fitness scores (by a domain expert) is necessary for the prediction of an outage.

Acknowledgements The authors would like to thank $3 \mathrm{E}$ (http://www.3e.eu) for granting access to the data and providing domain expert feedback on the results and Pierre Dagnely for the initial preprocessing of the data. Part of this work was subsidised by the Region of Bruxelles-Capitale - Innoviris.

\section{References}

1. van der Aalst, W., Adriansyah, A., van Dongen, B.: Replaying history on process models for conformance checking and performance analysis. WIREs Data Mining and Knowledge Discovery 2(2), 182-192 (2012). DOI 10.1002/widm.1045. URL https://onlinelibrary.wiley.com/doi/abs/10.1002/widm.1045

2. Van der Aalst, W., Weijters, T., Maruster, L.: Workflow mining: Discovering process models from event logs. IEEE transactions on knowledge and data engineering 16(9), 1128-1142 (2004)

3. van der Aalst, W.M.: Process Mining in the Large: A Tutorial, pp. 33-76. Springer International Publishing, Cham (2014). DOI 10.1007/978-3-319-05461-2_2. URL https : //doi.org/10.1007/978-3-319-05461-2_2

4. Bolt, A., de Leoni, M., van der Aalst, W.M.P.: A visual approach to spot statisticallysignificant differences in event logs based on process metrics. In: S. Nurcan, P. Soffer, M. Bajec, J. Eder (eds.) Advanced Information Systems Engineering, pp. 151-166. Springer International Publishing, Cham (2016)

5. Calderón-Ruiz, G., Sepúlveda, M.: Automatic discovery of failures in business processes using process mining techniques. In: Anais Principais do IX Simpósio Brasileiro de Sistemas de Informação, pp. 439-450. SBC (2013)

6. Dagnely, P., Tsiporkova, E., Tourwé, T., Ruette, T., De Brabandere, K., Assiandi, F.: A semantic model of events for integrating photovoltaic monitoring data. In: 2015 IEEE 13th International Conference on Industrial Informatics (INDIN), pp. 24-30 (2015). DOI 10.1109/INDIN.2015.7281705

7. van Dongen, B.F., de Medeiros, A.K.A., Verbeek, H.M.W., Weijters, A.J.M.M., van der Aalst, W.M.P.: The prom framework: A new era in process mining tool support. In: Proceedings of the 26th International Conference on Applications and Theory of Petri Nets, ICATPN?05, p. 444?454. Springer-Verlag, Berlin, Heidelberg (2005). DOI 10. 1007/11494744_25. URL https://doi.org/10.1007/11494744_25

8. Gupta, N., Anand, K., Sureka, A.: Pariket: Mining business process logs for root cause analysis of anomalous incidents. In: W. Chu, S. Kikuchi, S. Bhalla (eds.) Databases in Networked Information Systems, pp. 244-263. Springer International Publishing, Cham (2015)

9. Leemans, S.J., Fahland, D., van der Aalst, W.M.: Discovering block-structured process models from incomplete event logs. In: International Conference on Applications and Theory of Petri Nets and Concurrency, pp. 91-110. Springer (2014) 
10. Leemans, S.J.J., Fahland, D., van der Aalst, W.M.P.: Process and deviation exploration with inductive visual miner. In: BPM (Demos), CEUR Workshop Proceedings, vol. 1295 , p. 46. CEUR-WS.org (2014)

11. Rozinat, A., van der Aalst, W.M.P.: Conformance checking of processes based on monitoring real behavior. Inf. Syst. 33(1), 64-95 (2008). DOI 10.1016/j.is.2007.07.001. URL http://dx.doi.org/10.1016/j.is.2007.07.001

12. Suriadi, S., Ouyang, C., van der Aalst, W.M.P., ter Hofstede, A.H.M.: Root cause analysis with enriched process logs. In: M. La Rosa, P. Soffer (eds.) Business Process Management Workshops, pp. 174-186. Springer Berlin Heidelberg, Berlin, Heidelberg (2013)

13. van der Aalst, W., de Medeiros, A.: Process mining and security: Detecting anomalous process executions and checking process conformance. Electronic Notes in Theoretical Computer Science 121, 3 - 21 (2005). DOI https://doi.org/10.1016/j.entcs.2004.10. 013. URL http://www.sciencedirect.com/science/article/pii/S1571066105000228 Proceedings of the 2nd International Workshop on Security Issues with Petri Nets and other Computational Models (WISP 2004)

14. Weijters, A., van Der Aalst, W.M., De Medeiros, A.A.: Process mining with the heuristics miner-algorithm. Technische Universiteit Eindhoven, Tech. Rep. WP 166, 1-34 (2006)

15. Wilson, P., Dell, L., Anderson, G.: Root Cause Analysis: A Tool for Total Quality Management Workbook. ASQC Quality Press (1993). URL https://books.google. be/books?id=36S086CQ2CYC 